\title{
Límites y contradicciones de la razón pura, entre la Dissertatio (1770) y la Crítica de la razón pura (1781)
}

\author{
Boundaries and contradictions of pure reason, between the \\ Dissertatio (1770) and the Critique of pure reason (1781)
}

Fernando Moledo

fernandomoledo@gmail.com

(Universidad de Buenos Aires/CONICET, Buenos Aires, Argentina)

\begin{abstract}
Resumen: En este artículo sostengo que es posible detectar un estadio específico en la génesis de la Antinomia de la razón pura. Dicho estadio se encuentra entre 1770 y 1781 y consiste en un disenso que tiene lugar dentro de la razón, entre las funciones que en la KrV serán atribuidas al entendimiento puro y a la razón pura en sentido estricto.
\end{abstract}

Palabras-clave: Kant; antinomia; razón; límites; metafísica.

\begin{abstract}
In this paper I claim that it is possible to find a particular stage in the development of the Antinomy of pure reason, understand as a conflict of reason whit itself. This stage is placed between 1770 and 1781 and consists in a conflict which arises in reason itself but between the functions that later will be assigned to pure understanding and pure reason in strict sense.
\end{abstract}

Keywords: Kant; antinomy; reason; boundaries; metaphysics.

\section{Introducción}

En la Crítica de la razón pura $(=\mathrm{KrV})^{1}$ Kant define la Antinomia de la razón pura como un "conflicto de la razón" (A 408 / B 435) o "disenso de la razón consigo misma" (A 464 / B 492). Este disenso ocurre cuando la razón, entendida específicamente como facultad de conocimiento de lo incondicionado, se refiere a los fenómenos

\footnotetext{
1 Todas las referencias a la obra de Kant se toman de la Edición Académica de la obra de Kant = AA (Kant, Immanuel, Gesammelte Schriften Hrsg.: Bd. 1-22 Preussische Akademie der Wissenschaften, Bd. 23 Deutsche Akademie der Wissenschaften zu Berlin, ab Bd. 24 Akademie der Wissenschaften zu Göttingen. Berlin 1900ss.) y se citan siguiendo las siglas y las convenciones provistas por la revista Kant-Studien. En el caso de la Crítica de la razón pura, como es habitual se indica el número de página de las ediciones originales de Riga de 1781 y 1787 por medio de las letras A y B, respectivamente. Para las traducciones de la Crítica de la razón pura sigo la traducción de Mario Caimi: Kant (2009). El resto de las traducciones son propias.
} 
y concibe la totalidad incondicionada de sus condiciones. El origen del disenso en cuestión se encuentra en el hecho de que la serie regresiva de las condiciones de los fenómenos puede representarse como una totalidad incondicionada de dos maneras contrapuestas: como una serie que posee un primer miembro que le da comienzo y como una serie que no posee un primer miembro. Esta peculiaridad da lugar a conceptos puros de la razón, a los que Kant llama ideas trascendentales, en base a los cuales son formulados juicios contradictorios acerca del mundo, como totalidad de los fenómenos. Concretamente, da lugar a cuatro pares de juicios contradictorios, que expresan un "conflicto de las ideas trascendentales" (A 426 s. / B 454 s.); por ejemplo - según el primero de esos cuatro conflictos - a los juicios: "El mundo tiene un comienzo en el tiempo, y en el espacio también está encerrado en límites” (A 426 I B 454) y "El mundo no tiene comienzo, ni límites en el espacio, sino que es infinito tanto en lo que respecta al tiempo, como en lo que respecta al espacio" (A 425 / B 455). ${ }^{2}$

Como ha observado Norbert Hinske, la noción de un conflicto que tiene lugar en la razón misma i. e. la noción de un conflicto de la razón consigo misma, constituye la etapa ulterior de la evolución de un problema del que Kant se ocupa desde el primero de sus escritos. Este problema ha sido definido por Hinske, en términos generales, como el problema antinómico: se trata del hecho que la metafísica, entendida como una ciencia racional pura, desemboca en contradicciones cuando no

2 Estos juicios contradictorios producidos por la razón, que Kant detalla en la Antitética de la razón pura, exhiben, en cada caso, principios racionales cosmológicos o leyes racionales acerca del mundo: dicen cómo debemos pensar el mundo y pretenden proporcionar conocimiento sobre él. En ese sentido se los puede pensar como leyes. Precisamente por eso Kant define la Antinomia de la razón pura de manera más exacta como un "conflicto de las leyes (...) de la razón pura" (A 407 / B 434). La definición de la Antinomia, como la de un conflicto que tiene lugar dentro de la razón, y que se plantea entre las leyes de esa facultad, es la definición que será recogida por Samuel Albert Mellin (1797, p.287) y por Erhard Schmid (1795, p.59) en sus respectivos diccionarios de la filosofía kantiana y puede ser considerada, por eso, como la definición de la Antinomia de la razón pura en sentido estricto. La idea de una "antinomia" como un conflicto de leyes puede deber su origen a Baumgarten, que en su Initia Philosophiae practicae acroamatice scripsit afirma: "Leges morales oppositae colliduntur, earumque collisio est ANTINOMIA. Cumque contradictio vel sit vera, vel apparens, antinomiae erunt vel verae, vel apparentes. Ad contradictionem veram requiritur accurate eiusdem" (Baumgarten, 1760, § 85, AA, 19: 40 - 41). Hinske, que es quien hace este señalamiento respecto del origen del término antinomia, entendido como un conflicto de leyes, advierte que la novedad que introduce Kant al respecto consiste, precisamente, en entender la Antinomia como un conflicto de leyes de la razón consigo misma (Hinske, 1980). Pero ¿cuáles son esas leyes? Para Heimsoeth, ellas son los juicios puros que brotan de la razón y que son tematizados en la sección "Antitética de la razón pura" (Heimsoeth, 1967, p.199). Pero Hinske ha discutido esta interpretación, argumentando que las leyes que conforman el conflicto de la razón consigo misma serían el origen mismo de la antitética i. e. de los juicios contradictorios de la razón. Dichas leyes consistirían concretamente, en lo siguiente: "por un lado, [en] la ley de anclar todo lo condicionado finalmente en algo incondicionado, por el otro lado [en] la exigencia de ver, en el ámbito de la experiencia, cada condición nuevamente como condicionada (...) por un lado [se trata] de la razón en sí misma, de la razón 'pura', por el otro, en cambio, [se trata] de la razón respecto de la experiencia y de la elaboración de ella [i. e. la experiencia] por el entendimiento" (Hinske, 1965, p.492). Mi interpretación sigue de cerca la propuesta de Hinske. 
la precede un examen crítico de su validez (Hinske,1970, pp.106 y ss, Hinske, 1965). En ese sentido, este autor ha señalado que la etapa de la evolución del problema antinómico inmediatamente anterior al planteo definitivo de él que tiene lugar en la KrV consiste en el planteo de un disenso entre facultades que se encuentra en la disertación inaugural de 1770, Sobre la forma y los principios del mundo sensible e inteligible (de aquí en más: Dissertatio): el "disenso entre la facultad sensitiva y la intelectual" (MSI AA 2: 389).

Ciertamente, el señalamiento de Hinske ha aportado un valioso elemento para la comprensión de la evolución del pensamiento kantiano en general y de la génesis de la $\mathrm{KrV}$ en particular. Ahora bien, un grupo de anotaciones manuscritas (Reflexiones) de Kant posteriores a 1770 y anteriores a la publicación de la KrV en 1781, permiten detectar el planteo de un disenso de otro tipo, más complejo que el de 1770, aunque todavía distinto del que tendrá lugar en 1781, y que por eso puede ser situado entre ambos. En este artículo voy a estudiar ese disenso con el propósito de mostrar que, efectivamente, constituye una etapa específica de la evolución del problema antinómico, que se encuentra entre 1770 y 1781 . Dicha etapa - sostendré - se caracteriza por el hecho de que el disenso tematizado originalmente en la Dissertatio como un disenso que tiene lugar entre dos facultades (la facultad sensitiva y la intelectual) se traslada ahora al intelecto, entendido como facultad superior de conocimiento en general. Sin embargo - argumentaré - que estudiaremos no se plantea todavía como un disenso de la razón consigo misma, entendida específicamente como facultad de conocimiento de lo incondicionado. En cambio, él se plantea como un conflicto que tiene lugar en el intelecto o facultad superior de conocimiento en general, entre las funciones de conocimiento puro que luego, en la KrV, serán atribuidas específicamente al entendimiento puro (como facultad que da principios puros sobre los objetos de experiencia) y a la razón pura en sentido estricto (como facultad que proporciona principios sobre lo incondicionado).

Para lograr nuestro propósito, (1) en la primera sección de este artículo estudiaremos la concepción kantiana de los límites de la razón. Tras ello, (2) en la segunda sección, estudiaremos el disenso entre la facultad sensitiva y la intelectual tematizado en la Dissertatio. Luego, (3) nos concentraremos en el desarrollo posterior de esa doctrina; específicamente, vamos a poner el foco de la atención en la noción de “concepto-límite”. (4) Finalmente, a partir de los elementos discutidos, estudiaremos las Reflexiones posteriores a 1770 en las que Kant se refiere por primera vez a las contradicciones de la razón, como producto de un conflicto que tiene lugar entre los principios de conocimiento puro acerca de los fenómenos basados en las categorías y aquellos que brotan de los límites que la razón pone a la serie originada por esos principios. 


\section{Los límites de la sensibilidad y de la razón}

Muy poco tiempo después de haber presentado la disertación de 1770 Kant le anuncia a Marcus Herz, en una carta del 7 de junio de 1771, que trabaja en una obra nueva, cuyo título será Los límites de la sensibilidad y de la razón (Die Grenzen der Sinnlichkeit und der Vernunft) (Brief AA 10: 123). Este proyecto, sin embargo, será reemplazado rápidamente por otro. En efecto, en la conocida carta que le envía a Herz el 21 de febrero de 1772, Kant le anuncia que ahora trabaja en una obra nueva, que tendrá por título Crítica de la razón pura (Brief AA 10: 132). Pero ¿a qué pudo haberse referido Kant con el título que piensa para la obra que finalmente decide no escribir: Los límites de la sensibilidad y de la razón? ¿Cuáles son los límites mencionados? Eso es justamente lo que nos interesa indagar ahora y aquello que constituye el punto de partida de nuestra investigación.

Por supuesto, no es posible dar una respuesta definitiva a la pregunta que nos acabamos de plantear, pero podemos ensayar una interpretación verosímil a partir del examen de la propia Dissertatio. Comencemos por los "límites de la sensibilidad" a los que se refiere Kant en el título de la obra nunca escrita. Si consideramos la Dissertatio parece fácil determinar qué pudo haber tenido en mente Kant a la hora de referirse a ellos. En efecto, no es difícil identificar esos límites con las formas de la sensibilidad que son tematizadas por primera vez en la Dissertatio, y que serán recogidas años después en la Estética trascendental: las intuiciones puras del espacio y del espacio. Veamos.

Ciertamente, debido a que las intuiciones puras del espacio y del tiempo son las formas de la sensibilidad, los objetos, en la medida en que son conocidos sensiblemente, serán conocidos sólo como fenómenos, pero no como cosas en sí mismas. En efecto, por más generalizaciones que se hagan mediante el intelecto afirma Kant al respecto - lo que conozcamos sensiblemente estará siempre sujeto a las formas de la sensibilidad; por eso será siempre conocimiento fenoménico y nunca conocimiento de las cosas tal como son en sí mismas. Éste último - agrega Kant en abierta diferencia de lo que sostendrá años más tarde en la KrV - sólo se logra gracias a los conceptos puros proporcionados por el "uso real" del intelecto. Pues estos conceptos, por originarse con entera independencia de la sensibilidad, a partir de la mera actividad del intelecto, ${ }^{3}$ servirían para conocer efectivamente cómo son las cosas en sí mismas, con independencia de su apariencia sensible (MSI AA 2: 392 y ss.).

Está claro cuáles pudieron haber sido los aludidos límites de la sensibilidad: las intuiciones puras del espacio y del tiempo, en tanto que, como formas de la sensibilidad, limitan el conocimiento sensible a los fenómenos. Pero ¿cuáles son los

3 Sobre la doctrina kantiana introducida en la Dissertatio, acerca del origen de las representaciones puras, cf. Oberhausen (1997). 
“límites de la razón"? La respuesta a esta nueva pregunta no es tan fácil de lograr. Sin embargo, también es posible hacer un ensayo satisfactorio para ella, a partir de lo que Kant afirma en la Dissertatio. ${ }^{4}$

En la primera sección de la Dissertatio, Kant se refiere a los conceptos intelectuales de "lo simple” (MSI AA 2: 387) y “el mundo" (MSI AA2: 387). Lo interesante para nosotros es que a estos conceptos Kant los llama entonces, precisamente: “límites" (terminos) (MSI AA 2: 389). En efecto, se refiere a ellos como límites del análisis y de la síntesis, dos actividades que el intelecto puede llevar a cabo tomando como punto de partida el concepto puro de un compuesto sustancial. Estudiemos más de cerca este punto.

Un compuesto sustancial - sostiene Kant - es un todo formado de partes. Si partimos, pues, del concepto de un compuesto sustancial, entonces el regreso en el análisis no puede concluir sino en una parte que ya no forma un todo, esto es, en lo simple; y el progreso en la síntesis o composición, no puede finalizar sino en un todo que ya no es parte de ninguna otra cosa, esto es, en lo que se llama un mundo. Lo simple y el mundo son, pues, conceptos puros que surgen a partir de la necesidad que tiene la mente, respectivamente, de poner un límite al regreso en el análisis y al progreso en la síntesis, para poder "aquietarse" (MSI AA 2: 389).

En la Reflexión 4201, datada en torno a 1769 - 1770, encontramos la misma doctrina. Kant escribe lo siguiente:

El terminus de la coordinación por análisis, esto es, a priori, es lo simple; el terminus de ella por síntesis, esto es, a posteriori, es el mundo. El terminus de la subordinación a priori es la causa suprema, [el terminus de la subordinación] a posteriori [es] la totalidad de las consecuencias. La razón reconoce incompleta la compresión suya que no está encerrada en terminos (Refl. 4201 AA 17: 440).

Podemos pensar, pues, que los conceptos tematizados en la Dissertatio como límites que le sirven a la mente para aquietarse en el regreso analítico y en el progreso sintético - lo simple y el mundo - son los límites de la razón a los que se refiere Kant en el título de la obra nunca escrita.

Ahora bien, la doctrina acerca de los límites de la razón cumple un papel argumental decisivo en la Dissertatio precisamente porque le permite a Kant explicar y resolver el origen de las contradicciones que hacen imposible el progreso de la metafísica. Examinémoslo en la próxima sección.

4 Respecto del término "razón", por los límites de la cual se pregunta, cabe aclarar que en la Dissertatio Kant utiliza el término "Intelecto", asociado al de un uso real de él, pero también los términos "inteligencia" y "racionalidad", para referirse, de manera general y sin distinción, a la facultad superior de conocimiento i. e. la espontaneidad de la mente, en su totalidad, como facultad que nos sirve para conocer aquello que no puede ser conocido por medio de los sentidos. Las distinciones específicas entre el entendimiento puro, como facultad que proporciona los conceptos puros de un objeto en general y los principios puros generales del conocimiento empírico, y la razón pura (en sentido estricto), como facultad de conocimiento de lo incondicionado, que proporciona las ideas trascendentales y los principios puros sobre los incondicionado basados en ellas, es posterior a 1770. 


\section{El disenso entre la facultad sensitiva y la intelectual}

Las contradicciones que impidieron hasta ahora el progreso de la metafísica - argumenta Kant en la Dissertatio - son, en realidad, meramente subjetivas, y se originan en un disenso entre la facultad sensitiva y la intelectual. En efecto sostiene - cuando algo dado es pensado como un compuesto sustancial, el análisis, que retrocede del todo a las partes, y la síntesis, que progresa de las partes al todo, puede llevarse a cabo sensiblemente de manera infinita, pues dicho compuesto se encuentra en el tiempo (que es una magnitud continua infinita), y es posible pensarlo, por eso, como algo que dura y que debe existir en el instante siguiente y que, por lo tanto, es posible descomponer en partes o componer infinitamente (MSI AA 2: 388). La facultad sensitiva parece contradecir a la intelectual, pues, según lo que la primera enseña, ni lo simple, ni el mundo, parecerían ser posibles. ¿Cómo se resuelve el problema?

El problema se resuelve cuando se entiende que su origen es el tiempo, como condición subjetiva del conocimiento sensible. Pues eso quiere decir que todo lo que se afirme en base a esa condición subjetiva será plenamente válido para los fenómenos, pero que no dirá nada acerca de las cosas en sí mismas, tal como se las representa el intelecto puro con plena independencia de la sensibilidad, como cuando él produce los conceptos de lo simple y del mundo. Lo que parecía imposible objetivamente (lo simple y el mundo) era en realidad sólo una imposibilidad de carácter subjetivo, basada en el "disenso entre la facultad sensitiva y la intelectual" (MSI AA 2: 389) y que, por eso mismo, no tiene consecuencias para la metafísica, si a ésta se la entiende, correctamente, como "filosofía pura" (MSI 2: 411) i. e. como conocimiento puramente intelectual, enteramente independiente de la sensibilidad. ${ }^{5}$

Hemos estudiado la doctrina de los límites de la razón y hemos visto cómo ella le sirve a Kant para explicar y resolver las contradicciones de la metafísica, señalando que el origen de esas contradicciones es subjetivo y se encuentra en un disenso entre la facultad sensitiva y la intelectual. Ahora pasemos al análisis de la doctrina sobre los límites de la razón y de su relación con las contradicciones que se presentan en la metafísica, según el desarrollo de esa doctrina que tiene lugar con posterioridad a 1770 .

\section{Conceptos-límite}

Reparemos en la sección dedicada a la cosmología que se encuentra en la lección sobre metafísica datada en el período 1775 - 1780 y que está recogida en el

5 Sobre este punto puede verse un análisis más detallado en mi artículo: Moledo (2016). 
manuscrito conocido como L1. ${ }^{6}$ En efecto, en ella es posible encontrar un desarrollo más complejo de la doctrina de los límites de la razón.

La razón - explica Kant en la lección - tiene necesidad de poner un límite en la síntesis determinada por los conceptos puros del entendimiento. Estos conceptos son aquellos que más tarde, en la KrV, serán recogidos en la tabla de las categorías, bajo el título de "Relación": los conceptos puros de la relación de los accidentes con la sustancia, del fundamento y lo fundado, y de (la acción recíproca de) las partes en un todo. Los límites a esa síntesis - afirma - son puestos por medio de conceptos a los que Kant se refiere ahora directamente como "conceptos-límite” (Grenzbegriffe). Estos conceptos-límite son: el concepto de lo sustancial, en la relación de los accidentes con la sustancia; el concepto de la causa de todos los efectos, que al mismo tiempo no es efecto de nada, esto es, el concepto de Dios, en la relación de causa y efecto; y el todo que ya no forma parte de ninguna totalidad, esto es, el concepto de mundo, en la relación de las partes en un todo. Concretamente, podemos leer al respecto lo siguiente:

Ya hemos hablando en la ontología sobre los conceptos-límite, que constituyen el límite en la serie del conocimiento. - En la relación estaban estos tres conceptos: la relación de la sustancia con el accidente, de la causa con el efecto, y de las partes con el todo. - En todos estos conocimientos podemos pensar un primero y un último, por medio de los cuales, en estos conocimientos, se produce una completudo o totalidad. - En la relación de la sustancia con el accidente lo sustancial es aquello que ya no es más accidente de otro. - En la relación de la causa con el efecto, el concepto-límite es la causa primera, que no es causatum alterius. - En la tercera relación, [la relación] del todo con las partes, el concepto-límite es aquel todo, que ya no es más parte de otro, y ese es el concepto del mundo. Nuestra razón tiene la necesidad, de que ella no está sosegada, hasta que encuentra una completudinem en la serie de las cosas o hasta que puede pensar una totalidad completa ( $V$ - Meth/ L1. AA 28: 195).

De acuerdo con este pasaje, es posible constatar que entre 1770 y el momento en el que imparte la lección de metafísica recogida en el manuscrito L1 (presumiblemente, comienzos de la segunda mitad de la década de 1770) Kant desarrolla la doctrina sobre los límites que la razón pone a la síntesis determinada por los conceptos puros del entendimiento -doctrina que había planteado originalmente en la Dissertatio introduciendo para ello la noción de concepto-límite.

Ahora bien, para nosotros, lo especialmente interesante al respecto, es poder constatar, además, el hecho de que Kant se sirve ahora de esta doctrina para explicar el origen de un conflicto o de un disenso más complejo que el que de la Dissertatio, y que es señalado ahora como origen de las contradicciones planteadas en metafísica, que habían sido tematizadas anteriormente. Este disenso ya no tiene lugar entre la

6 Para el origen y datación de la lección sobre metafísica recogida en el manuscrito L1, cf. Heinze (1894, p.516) y Naragon (2000). 
facultad sensitiva y la intelectual, como en 1770, esto es, entre dos facultades o fuentes de conocimiento distintas. En cambio, se trata ahora de un disenso que se desplaza al interior mismo del intelecto, como facultad superior de conocimiento o facultad de pensar en general, donde se plantea como un conflicto que tiene lugar entre la función sintética que más tarde será distinguida específicamente y asignada al entendimiento puro y la que será referida a la razón pura en sentido estricto i. e. entendida como facultad de conocimiento de lo incondicionado. Pero veámoslo en detalle a continuación.

\section{Conceptos-límite y contradicciones de la razón}

Antes que nada, hagamos una aclaración acerca de la fuente de la que nos vamos a servir a continuación. Esa fuente es un conjunto de Reflexiones que originalmente Adickes (1925, AA 14: xxxviii) refiere a la fase k, fase que sitúa alrededor de 1769, pero del que luego se ha mostrado que puede contener anotaciones posteriores a $1770 .^{7}$ Y es así, como posteriores a 1770 , como vamos a considerar a las Reflexiones que analizaremos aquí. Dicho esto, pasemos, ahora sí, a la primera de ellas que examinaremos: la Reflexión 4033.

En la Reflexión mencionada Kant anota lo siguiente:

El concepto de lo necesario es no obstante, en primer lugar, un concepto dado por la razón, porque sólo por medio de él algo puede ser determinado. La necesidad absoluta es un concepto-límite, porque sin él no habría completudo en la serie de lo contingente (Refl. 4033 AA 17: 392).

Esta Reflexión recoge la doctrina que estuvimos analizando hasta aquí y que Kant expresa, como lo hará más tarde en la lección de metafísica L1, por medio de la noción de concepto-límite. Pues bien, lo importante es que, como adelantamos, siguiendo esa línea argumental, Kant plantea aquí una nueva forma de disenso que tiene lugar ahora dentro de la facultad intelectual misma. Ello ocurre en la Reflexión 3928, de la misma fase.

En esta Reflexión, Kant se refiere a la necesidad de poner un límite a la síntesis de la relación de subordinación o dependencia (esto es, a la relación del fundamento y lo fundado). El principio formal de la razón, referido a la conexión real "todo lo que sucede tiene un fundamento determinante" -afirma Kant - determina una serie infinita, a la cual, a su vez, la razón necesita poner un límite para poder representarse esa serie de manera completa. De ello surge el principio: "todo tiene un primer fundamento".

Ahora bien, entre ambos principios se plantea, claramente, un problema.

7 Hinske (1970, pp.106 y ss), Hinske (1965). También Klemme sostuvo luego un punto de vista similar. Cf. Klemme (1996, p.41 n.). 
Pues, en efecto, en base a ellos, - sostiene Kant -, no se puede entender que la serie que el primer principio hace necesaria carezca de comienzo, pero tampoco se puede comprender cómo pueda ser que tenga lugar un comienzo en esa serie, que el primer principio hace infinita. La serie debe tener, pues, un comienzo y, al mismo tiempo, no tenerlo. En la Reflexión Kant anota, concretamente, lo siguiente:

Por lo que concierne al nexum realem, los principia materialia de él son las experiencias, los principia formales son: todo lo que sucede tiene un fundamento determinante $y$, segundo: todo tiene un primer fundamento. Estos principios son, ambos, sintéticos, aquél, para el uso de nuestra razón, éste para el término de ese uso. Pues según aquél vemos siempre, en la serie de las causas mutuamente determinantes, siempre hacia el fundamento más alto; y, según éste, admitimos que esa serie sea limitada. Pero es tan imposible representarse una serie de fundamentos subordinados, que no tiene principio, como concebir cómo ella comience (Refl. 3928 AA 17: 350 - 351).

Claramente, el conflicto del que se trata en esta Reflexión ya no es - como adelantamos - un mero disenso entre la facultad sensitiva y la intelectual. Se trata aquí, en cambio, de un disenso que se ha trasladado al seno mismo del intelecto o de la razón, entendida ésta última todavía en términos generales, como facultad de conocimiento superior o espontaneidad de la mente, y que tiene lugar entre las funciones de que más adelante serán referidas, específicamente, al entendimiento puro (dar principios sintéticos puros a los objetos de experiencia posible) y a la razón pura (dar principios sintéticos puros acerca de lo incondicionado).

Pues bien, en otra Reflexión de la misma fase k encontramos un punto de vista similar: nuevamente se plantea aquí un conflicto que surge partir de los principios racionales que determinan una serie sintética infinita (y que más tarde serán referidos específicamente al entendimiento puro) y los que surgen de la necesidad de poner un límite a esa serie para poder representársela de manera completa (que más tarde serán referidos a la razón pura en sentido estricto, como facultad de conocimiento incondicionado). Pero la novedad que nos encontramos ahora es que Kant se refiere a este conflicto como origen de las "contradicciones de la razón pura". Se trata de la Reflexión 3999, en la que leemos:

Las contradicciones de la razón pura surgen porque ella no obtiene sus proposiciones universales de lo particular, como la filosofía empírica, sino que juzga universalmente por concepto puros, pero al mismo tiempo requiere un terminum de la subordinación o de la coordinación, el cual contradice la universalidad (Refl. 3999 AA 17: 381). ${ }^{8}$

8 Cuáles son exáctamente las contradicciones de la razón que Kant tiene en mente aquí es algo muy difícil de determinar con plena seguridad en base a las Reflexiones de este período. De cualquier modo, y como lo ha señalado Hinske $(1998$, p.95 n) y lo ha observado también Schmitz (1998, p.66.), las afirmaciones que conforman las contradicciones de la razón tematizadas en la KrV se encuentran formuladas, en su totalidad, ya en distintas obras del período pre-critico de la evolución del pensamiento de Kant (cf. GSK AA 1: 156, NTH AA 1: 322 nota, 1: 314; PND AA 1: 400 y ss; MoPh AA 1: 477.) 
Evidentemente, nos encontramos aquí con una doctrina novedosa respecto de la que habíamos podido encontrar en 1770. Pasemos a las conclusiones que podemos sacar en base a ello.

\section{Consideraciones finales}

En Reflexiones de la fase k, encontramos la tematización de un conflicto que tiene lugar en el intelecto mismo, entendido en general como facultad de conocimiento superior, y que se produce entre las funciones que luego serán atribuidas de manera diferenciada, respectivamente, al entendimiento puro y a la razón pura (en sentido estricto, como facultad de conocimiento de lo incondicionado). Como hemos visto, Kant señala además que este conflicto es origen de las contradicciones de la razón pura i. e. de aquellas contradicciones a cuyo estudio Kant se dedica desde el comienzo de su actividad filosófica y que constituye el impedimento que hace imposible, hasta su resolución definitiva en la $\mathrm{KrV}$, el progreso de la metafísica.

Si bien el conflicto planteado en las Reflexiones que estudiamos no es el conflicto de la razón consigo misma al que Kant se refiere en la KrV, ciertamente constituye un desarrollo novedoso frente al planteo de la Dissertatio. Pues en efecto, ya no se trata de un disenso entre la facultad sensitiva y la intelectual, sino de un conflicto que se ha trasladado por completo al intelecto o facultad superior de conocimiento. De ello se puede concluir, como pretendíamos, que este conflicto constituye una nueva etapa, específicamente diferenciada, en la evolución que conduce a la Antinomia de la razón pura, entendida como un conflicto o disenso de la razón consigo misma; una etapa, pues, intermedia, que se encuentra entre la Dissertatio y la KrV. De este modo esperamos haber logrado contribuir a una mejor comprensión de la evolución del problema antinómico en particular, y de la génesis de la KrV en general.

\section{Referencias}

Adickes, E. (1925). „Einleitung in die Abtheilung des Handschriftlichen Nachlasses“. En AA: 14.

Baumgarten A. G. (1760). Initia Philosophiae practicae acroamatice scripsit: Halle.

Heimsoeth, H. (1967). Transzendentale Dialektik. Ein kommentar zu Kants Kritik der reinen Vernunft. Zweiter Teil. Berlin: Walter de Gruyter \& Co.

Heinze, M (1894). Vorlesungen Kants über Metaphysik aus drei Semestern. Abhandlungen der königlich Sächsischen Gesellschaft der Wissenschaften. 34. Band. Des XIV. Bandes der Abhandlungen der philosophisch-historischen Classe der Königl. Sächsischen Gesellschaft der Wissenschaften No. VI.: Leipzig.

Hinske, N. (1965). Kants Begriff der Antinomie und die Ettappen seiner Ausarbeitung. Kant-Studien, 56, pp.485-496. 
- (1970). Kants Weg zur Transzendentalphilosophie. Der dreißigjährige Kant. W. Stuttgart - Berlin - Köln - Mainz: Kohlhammer Verlag.

. (1980). „Reimarus zwischen Kant und Wolff“. En Borinski, L. und Walter, W. (Hrgb.). Logik im Zeitalter der Aufklärung. Studien zur „Vernunftlehre“ von Hermann Samuel Reimarus. Göttingen: Vandenhoeck \& Ruprecht.

Kant, I. (1900 y ss = AA). Gesammelte Schriften Hrsg.: Bd. 1-22 Königlich Preussische Akademie der Wissenschaften, Bd. 23 Deutsche Akademie der Wissenschaften zu Berlin, ab Bd. 24 Akademie der Wissenschaften zu Göttingen: Berlin.

- (2009). Crítica de la razón pura. Traducción, notas e introducción de Mario Caimi. Buenos Aires: Colihue.

Klemme, H. F. (1996). Kants Philosophie des Subjekts. Systematische und Entwicklungsgeschichtliche Untersuchungen zum Verhältnis von Selbstbewußtsein und Selbsterkenntnis. Hamburg: Meiner.

Mellin, G. S. A. (1797). Enzyclopädisches Wörterbuch der kritischen Philosophie. Erster Band: Züllichau und Leipzig.

Moledo, F. (2016). Die neue Auffassung der Metaphysik als reine Philosophie in der Inauguraldissertation und ihre propädeutische Bedeutung im Rahmen der Entwicklungsgeschichte der KrV. Kant-Studien, 107, pp.485-495.

Naragon, S. (2000). The Metaphysics Lectures in the Academy Edition of Kant's gesammmelte Schriften. Kant-Studien, 91, pp.189-215.

Oberhausen, M. (1997). Das neue apriori. Kants Lehre von einer „ursprünglichen Erwerbung " apriorischer Vorstellungen. Forschungen und Materialien zur deutschen Aufklärung (FMDA) herausgegeben von Norbert Hinske. FrommannHolzboog: Stuttgart - Bad Canstatt.

Schmid, C. C. E. (1795). Wörterbuch zum leichtern Gebrauch der Kantischen Schriften nebst einer Abhandlung: Jena.

Schmitz, H. (1989). Was wollte Kant? Bonn: Bouvier Verlag. 